\title{
Panorama urgente sobre la mediatización de la circulación y algunos de sus estudios
}

Resumen: En este artículo se recupera la noción de circulación desde su apropiación de la teoría marxiana por parte de dos autores referenciales en las investigaciones en comunicación en Europa y América Latina (S. Hall y E. Verón) en los inicios de los años '80, hasta su actual revalorización por parte de autores que se interesan por la mediatización en las sociedades contemporáneas, en las que las redes y los cambios tecnológicos, culturales y discursivos han transformado las condiciones de circulación.

Palabras clave: Circulación. Mediatización. Contemporaneidad. Redes.

\section{Panorama urgente sobre a midiatização da circulação e alguns de seus estudos}

Resumo: Neste artigo, a noção de circulação é recuperada de sua apropriação da teoria marxiana por dois autores referenciais na pesquisa em comunicação na Europa e na América Latina (S. Hall e E. Verón) no início dos anos 1980, até sua atual reavaliação por autores que estão interessados na midiatização nas sociedades contemporâneas, em que as redes e mudanças tecnológicas, culturais e discursivas transformaram as condições de circulação.

Palavras-chave: Circulação. Midiatização. Contemporaneidade. Redes.

\section{Urgent panorama on the mediatization of circulation and some of its studies}

\begin{abstract}
In this article, the notion of circulation is recovered from its appropriation of Marxian theory by two referential authors in communications research in Europe and Latin America (S. Hall and
\end{abstract}

Gastón Cingolani ${ }^{1}$

Professor-pesquisador Universidad Nacional de las Artes

(UNA)

E-mail: gastoncingolani@gmail.com 


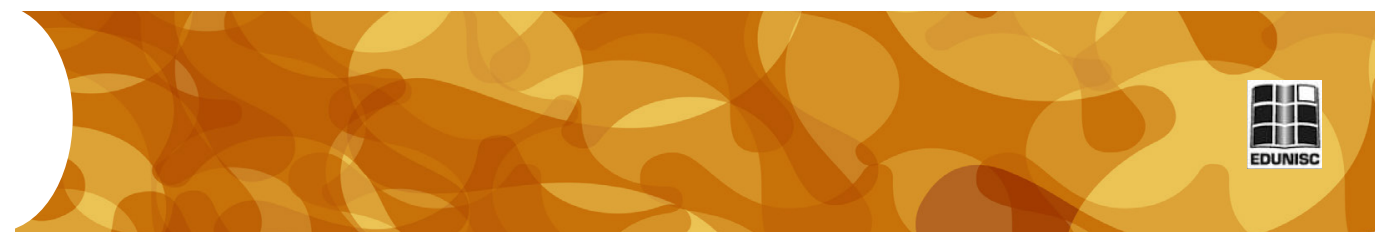

E. Verón) in the early 1980 s, up to its current revaluation by authors who are interested in mediating in contemporary societies, in which networks and technological, cultural and discursive changes have transformed the conditions of circulation.

Keywords: Circulation. Mediatization. Contemporaneity. Networks.

\section{Panorama urgente sobre la mediatización de la circulación y algunos de sus estudios}

Algunas circunstancias contemporáneas han vuelto a despertar el interés por la circulación en lo que se ha llamado (optimistamente) comunicación. A mediados del siglo anterior los estudios de comunicación se habían dividido - parecía que definitivamente entre aquellos que se dedicaban a la comunicación mediada por tecnologías de gran escala (hoy, mediatización) y los que se dedicaban a la comunicación producida en la inmediatez interpersonal. Eran poco considerados los casos de las comunicaciones interindividuales mediatizadas (el teléfono, el correo postal). Por entonces, aquellos estudios no preveían que con el cambio de siglo se iba a generar una inmensa autopista para el flujo de mensajes, que acerca enormemente los dos polos de esta división. Ese acercamiento no está hecho de otra cosa que de múltiples matices facilitados por las distintas interfaces, plataformas y dispositivos digitales en red, para producción y recepción de mensajes y contenidos de acceso público y/o privado, en diferentes escalas.

En la vieja división teórica, la circulación era (simplificando un poco) lineal en los casos de mediatización, y multidireccional en la interacción inmediata. Esto llevó a Winkin (1987) a distinguir ambos modelos como del "telégrafo y la orquesta". Entre algunos de los síntomas más salientes pero menos agradables de esa linealización estuvo la necesidad de introducir la noción de feedback (WEAVER, 1972; WIENER, 1972) y luego el debate (con el tiempo, devenido absurdo) sobre si la recepción era activa o pasiva.

El fortalecimiento del interés en dos rasgos que (a la larga) reestructuraron la cuestión, posibilitó que algunas teorías superaran mejor las crisis vertiginosas que ofrecen los cambios tecnológicos y culturales contemporáneos.

La noción de circulación se posiciona precisamente como clave, pero ¿en qué sentido? Para proponerlo en el final del artículo, antes debemos revisar qué se entiende y qué se entendió por circulación. 


\section{$\gamma_{\text {Rizoma }}$}

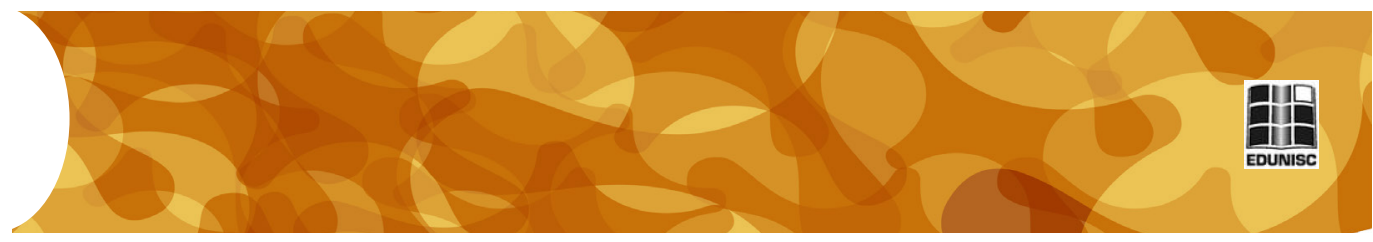

\section{Circulación no es distribución}

La noción de circulación en los estudios comunicacionales y mediáticos fue impulsada, con éxito diverso, tanto en Europa como en América Latina, de la mano de dos autores de gran protagonismo e influencia, respectivamente: Stuart Hall y Eliseo Verón. Hace unas cuatro décadas, escapando del modelo de emisión-recepción, ambos coincidían (quizás sin saberlo) en la recuperación de la noción de circulación del esquema nocional marxiano (producción-circulaciónconsumo), con semejanzas y diferencias en lo teórico. Desafiando frontalmente al modelo que suponía que la comunicación mediática se trata de una transferencia sin más de un mensaje desde un punto al otro, ambos proponían que en cada momento hay un trabajo de producción de sentido (VERÓN, 1988a), un "pasaje de formas" (HALL, 1980) y por tanto $-\mathrm{y}$ tal vez esta sea la coincidencia más importante- una asimetría (Hall), un desfase (Verón) en las significaciones producidas en las diferentes instancias. Coinciden también en que para conocer cómo funciona la comunicación mediática, es tan fundamental atender a los mensajes de los medios como a los efectos y lecturas que se producen en el ámbito de los receptores.

De esta revisión lo primero que merece atención es la potencial confusión entre circulación y “distribución de mensajes", al menos en lo que esa idea tiene de materialismo ingenuo. Tanto Hall como Verón habían comprendido que no es posible desligar la dimensión material de la significante, ni tampoco subsumir una a la otra. Por caso, la circulación requiere materialización, pero no es en sí misma material: se trata de circulación de sentido, y por tanto es una diferencia que surge del contraste entre materias significantes (llámense estos mensajes o discursos).

Esta noción de circulación como diferencia se desdibuja un poco en Hall, ya que la señala como un "momento" que no se distingue netamente del de la producción: pese a que parte de la necesidad de considerar como diferentes a cada uno de los momentos, solo sugiere una diferencia entre circulación y distribución, que no desarrolla (HALL, 1980, p. 117) ${ }^{2}$. La recuperación que Hall hace de la circulación concierne a la centralidad que ocupaban los medios en la etapa dominada por la mediatización masiva tradicional. Dos elementos subrayan este aspecto: el esquema, aún escapando del modelo emisión-recepción, ubica en su primer momento (el de la producción) a los medios, y la codificación está determinada por estos, mientras los consumidores son el último momento y su acción es la de decodificar. Es evidente que Hall otorga a la organización mediática un rango de dominancia como condicionante del sentido, estructuralmente marcada por la distribución broadcasting. ${ }^{3}$ El
2 Hall explicita haber recuperado esta noción de los Grundrisse y del tomo 1 de El Capital. Marx mismo no señala con nitidez los bordes de la circulación, como sí lo hace con las nociones de distribución y de cambio como partícipes del momento intermediario entre la producción y el consumo.

3 Hall desarrolla esta idea en el clásico texto sobre la televisión, en el seno de su interés por los fenómenos de la cultura de masas. 


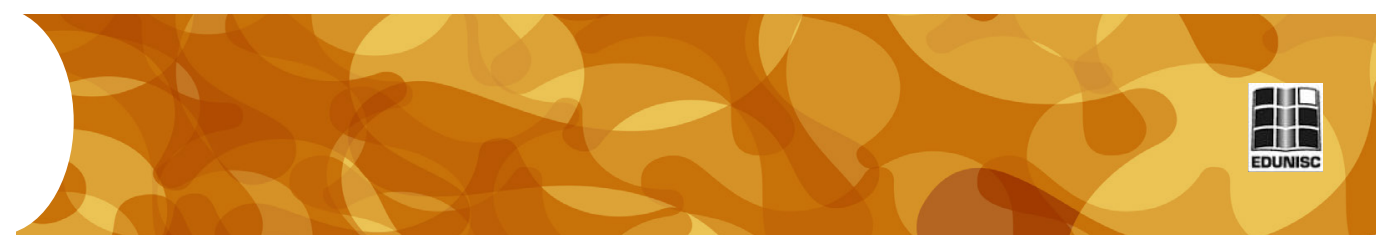

carácter diferencial de la circulación surge de la confrontación entre codificación y decodificación, ya que la segunda no se corresponde necesariamente con la primera. Posteriormente, quienes han estudiado problemas de circulación siguiendo esta línea conceptual culturalista, mantuvieron todo lo posible el rol de la centralización mediática incluso para explicar el asimétrico crecimiento y flujo cultural de las sociedades complejas - es el caso de Hannerz (1992).

En los trabajos de Verón, la relación entre materia y sentido está declinada diferente, lo que tiene consecuencia directa sobre la distinción entre circulación y distribución. La circulación sería la resultante de la diferencia de sentido entre la producción de un discurso y su reconocimiento; no se trata de un "momento" sino de un entre-dos, un intersticio, que puede comportar distancias temporales o espaciales, pero no necesariamente. Y de hecho, para Verón esa diferencia no es privativa del consumo de medios (VERÓN, 1988a; 1988b; 2001; 2004; 2013), sino que es constitutiva de toda la discursividad social. Este detalle amplifica su trascendencia si tomamos en cuenta no solo la obra de Verón desde sus inicios, sino también las consecuencias actuales a la hora de pensar las nuevas mediatizaciones.

Cuenta Verón en diferentes escritos que dio con el problema de la diferencia de sentido entre producción y reconocimiento trabajando con pacientes neuróticos, a comienzos de la década de 1970 con Carlos Sluzki, a propósito de la comunicación interindividual (SLUZKI, 1970; VERÓN, 1995; VERÓN, 2004). Aquellas investigaciones son explícitamente deudoras de los trabajos de Bateson, Watzlawick y la llamada Escuela de Palo Alto. Estaba claro que algunos postulados teóricos de estos se avenían mejor para comprender que la circulación no solo es un asunto diferencial, sino también de difícil observación para el actor social: si el sentido es opaco al propio sujeto, también lo es su desfase. "Todo ser humano conoce un margen de incertidumbre sobre el tipo de mensajes que emite; y todos necesitamos, finalmente, ver cómo se reciben nuestros mensajes para saber qué eran" (BATESON, 1987, p. 130).

Años más tarde, en investigaciones sobre mediatizaciones, Verón corroboró que este desfase de sentido se da tanto en procesos micro como macrosociales, aunque no de manera idéntica. Desde este punto de vista, el desfase expresa ni más ni menos que la circulación. Como sintetiza Sbardelotto (2018), "Sendo um processo circulatório, comunicação é aquilo que, mediante convergência de interação, desencadeia divergência de sentido”. ¿Pero para quién se hace visible la divergencia, el desfase, la asimetría?

La circulación se hace visible para un tercero, un observador que puede notar la diferencia. Esta teoría del observador es perseguida por Verón desde sus tempranos estudios lévistraussianos (VERÓN; 1961, p. 14-15) y su apropiación de los axiomas de Palo Alto. 
Según estas conceptualizaciones, en las que tienen lugar los contrastes poetizados con los símiles del "telégrafo y la orquesta" (WINKIN, 1987) o de las sociedades mecánicas y estadísticas (LéviStrauss), es viable pensar que la circulación es constitutiva de todo proceso comunicacional. (Aunque "comunicacional" sea un nombre optimista para eso que sucede cuando producimos un mensaje o cuando lo interpretamos, sin que tengamos idea de qué ha sucedido en la otra instancia).

\section{Circulación no es reproducción: asimetrías y desfases}

El término circulación tiene, lamentablemente, la reminiscencia de aquello que entra en una repetición, incluso que se transforma en circular, y por lo tanto supone algún tipo de retorno. Hall inicia su célebre texto recordando la crítica a la circularidad del modelo lineal emisor-mensaje-receptor: "Traditionally, mass-communications research has conceptualized the process of communication in terms of a circulation circuit or loop" (HALL, 2005, p. 117).

En su lectura marxiana, sin embargo y aun para confirmar la calamidad de los desequilibrios del industrialismo moderno proyectados en la comunicación, encontramos una hipótesis (que también tildamos de "optimista") de que habría algo de retroacción del consumo sobre la producción: "But the vast range must contain some degree of reciprocity between encoding and decoding moments, otherwise we could not speak of an effective communicative exchange at all" (HALL, 2005, p. 125).

Precisamente, en las (así llamadas) "comunicaciones masivas", no hay ni hubo jamás intercambios. Su optimismo reproductivista se suma al optimismo comunicacional, porque el célebre encoding/ decoding mantiene la idea -semiológica pero no semiótica, es decir, deudora de una conceptualización contenidista del sentido- de que impera un código, que en recepción podrá o no aceptarse.

La hipótesis del intercambio está basada en lo que Bateson (1987) y Watzlawick (WATZLAWICK; BAVELAS; JACKSON, 1981) llamaron puntuación de las secuencias de hechos, es decir, la visión de la comunicación como un proceso lineal, visión muy habitual en la conciencia individual: al mensaje de A le sigue la reacción o respuesta de $\mathrm{B}$, luego esta influye sobre el siguiente comportamiento de A y así sucesivamente. En las escenas cara a cara esto no sucede porque todo el tiempo estamos emitiendo y recibiendo señales: jamás se trata de un orden de turnos. En el extremo contrario, en las mediatizaciones tradicionales, a los medios masivos les costaba mucho obtener información concreta de lo que sucedía en el momento de la recepción, 


\section{$\gamma_{\text {Rizoma }}$}

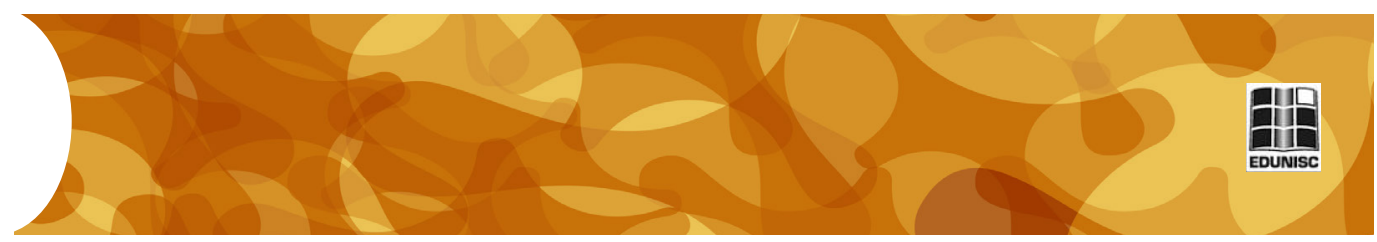

y a decir verdad, les importaba muy poco. Todo el ajuste intersubjetivo que podría haber en la interacción presencial, en las mediatizaciones de gran escala es cercano a cero.

Ahora bien, en clave culturalista, la circulación como reproducción no debe entenderse como un simple retorno al inicio, sino como una insistente confirmación de lo ya establecido. Pero en tal caso, es necesario recordar que la evolución de los sistemas de mediatizaciones estuvo en paralelo con la aceleración de las transformaciones socioculturales. Es lo que afirma Verón, pero es también la tesis de Hannerz (1992), que sostiene que la complejidad de las sociedades contemporáneas es resultado de sus asimetrías, y los medios solo acentúan esas asimetrías.

Los discursos producidos por las tecnologías y prácticas más características de la etapa de las mediatizaciones "masivas" o broadcasting no conservaban ni exponían en su superficie rastros de sus consumos ${ }^{4}$, y por lo tanto la circulación era opaca o invisible a la mirada de los actores sociales y a la de los propios medios, que solo la suponían. En ese sentido, es extraño que Hall sostuviera una hipótesis reproductivista, ya que según su esquema el momento del consumo daría lugar a que el sistema productivo reincorpore como feedback lo producido en la recepción.

Ahora bien, si este podía ser el momento de divergencia, ¿por qué insistir con nociones tan optimistas como reproducción o incluso, cultura, y no ver en la circulación la incertidumbre de la transformación? Verón (que investigó la recepción de los medios de manera empírica a lo largo de varias décadas, tanto en Argentina como en Francia) era bastante más pesimista al respecto, y encontraba que entre los medios y los consumidores podía llegar a haber algo así como universos paralelos, poco articulados, antes que un mismo sistema en franca retroalimentación. Esa desarticulación solo cosechaba una progresiva desconfianza de los consumidores respecto de los medios, reflejada también en múltiples trabajos tanto europeos como latinoamericanos (MARTÍN BARBERO, 1987; GARCÍA CANCLINI, 1995, MORLEY, 1996, LUHMANN, 2000; VERÓN, 2005; GROYS, 2008; SILVERSTONE, 2010), incluso en el marco de las teorías culturalistas. Al parecer, a los consumidores sí les interesaba saber qué sucedía en la recepción. Los propios investigadores de los cultural studies consideraron esto seriamente para comprender mejor lo que implicaba la recepción (DAYAN, 2000), y de hecho buscaron poner a prueba esta hipótesis (DAYAN, 1993).

Frente a esta paradoja, los estudios de inspiración veroniana parten de la premisa de que siempre hay circulación, siempre hay desfase; pero la mediatización es el proceso humano que acentuó la asimetría entre producción y reconocimiento, lo que se hizo
${ }^{4}$ El correo de lectores o el contestador telefónico en la radio, fueron avanzadas de un cambio; sin embargo, esas participaciones estaban sometidas a edición por parte de los mismos medios, lo que siempre propició más una fantasía de atención a sus audiencias que un rastro efectivo y fiable de su recepción. La mayor evidencia de esto la ofrece lo que comenzó a suceder con los portales de noticias que incluyen comentarios de lectores y usuarios, con cascadas de opiniones contrarias al mismo medio. 


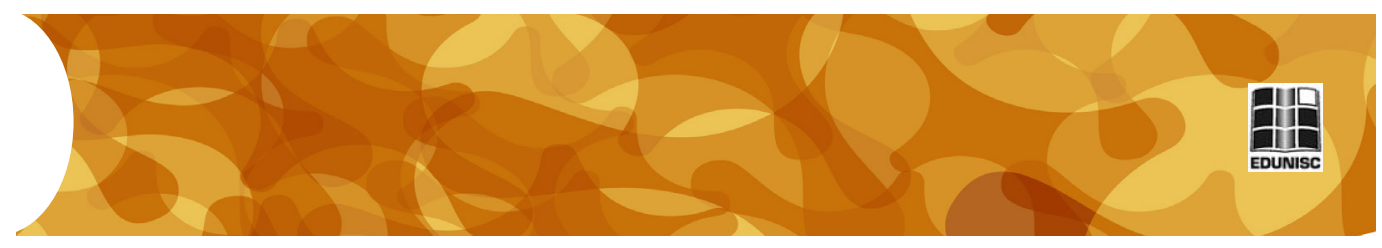

más evidente con sus materializaciones perdurables. Es esa línea semioantropológica sobre el largo trayecto de hominización, en la que Traversa (2017) subraya que la circulación mediatizada opera como proceso de descontextualización, es decir, de manifestación de las diferencias en la semiosis, principal (si no único) efecto que hace evolucionar a las culturas y a la especie, mediante dispositivos de materialización discursiva, entre ellos, quizás el más emblemático por su impacto transhistórico, el de la escritura. En consecuencia, si la mediatización broadcasting es un capítulo importante en un tipo de sociedad, las actuales redes ofrecen el desafío de otro capítulo, en el que pasamos de tratar con la circulación de la mediatización a la mediatización de la circulación, problema de dimensiones coyunturales pero también constitutivas de lo humano.

\section{Mediatización de la circulación: desafíos de la contemporaneidad}

Llegamos al fin a un momento que tiene algo de novedoso. Aquel "optimismo" de Hall hoy recupera sensatez, aunque sea por razones que quizás él no imaginó entonces: las tecnologías y prácticas actuales facilitan enormemente la información sobre lo que hacen, prefieren y producen los consumidores de los medios, pero también amplifican la conciencia de la circulación como diferencia. En la Red, no solo es muy difícil evitar los rastros, sino que también algunos de ellos nutren el trazado de las superficies mediáticas, haciéndose visibles para el propio usuario y para los demás, constituyendo un valor agregado, mezclándose con las producciones y huellas de otros individuos así como las de las instituciones mediáticas (CINGOLANI, 2017). Esa coexistencia no es una simple acumulación, ni se da igual en todas las partes. Desde el momento en que involucra procesos de mediatización (VERÓN, 2013; BRAGA, 2017), produce sentidos diferentes a los de la evanescencia de la interacción directa, in-mediata.

Hoy esta noción puramente diferencial de la circulación está siendo discutida (FERNÁNDEZ, 2018, p. 56-60), ya que las mediatizaciones nos ofrecen un nuevo panorama. Como sostiene Fausto Neto (2010), el pasaje de las "lógicas de los contratos" a las "lógicas de las interfaces", implica una circulación no solo como intersticio, sino también como "punto de articulación". Ninguna de las tecnologías de antaño se ha perdido, todas se reencuentran en plataformas y espacios multimediales y transmediales, y la circulación no es ya solo asunto de hacer coincidir las estrategias enunciativas de las instituciones mediáticas con consumidores estereotipados o asimilables a colectivos, sino también se busca conectar discursos y 


\section{$\gamma_{\text {Rizoma }}$}

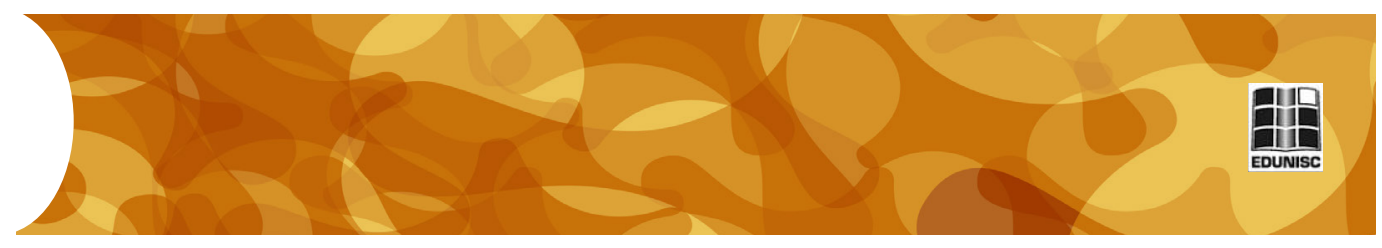

prácticas de y/o para individuos. Es lo que se busca sistematizar en varios trabajos actuales. Veamos algunos casos.

Sin deshacerse de la antigua conceptualización bipolar producción-recepción, Sbardelotto (2018) procura hacer ver la dinámica conmutable de estos polos, sobre todo en las redes. "Mas um interagente singular não pode ser produtor-receptor ao mesmo tempo em uma mesma ação comunicacional, nem apenas produtor ou receptor isoladamente: cada ação comunicacional ou cada interagente": esta idea no puede ser más cierta siempre que la comunicación sea pensada como interacción, y la unidad de su estudio siga siendo el agente. Pero esa conmutabilidad estaba planteada ya por los investigadores de la "Universidad invisible" de Palo Alto, que concebían a la conducta como comunicación en red (el símil de la orquesta es de Winkin), mucho antes de que las redes cibernéticas de uso cotidiano individual fueran diseñadas. No es este el avance conceptual esperable. De hecho, para Verón y su Teoría de la Discursividad, producción y reconocimiento no son polos o acciones intrínsecas a la semiosis, sino lugares asignados por el observador analista: todo discurso está en producción respecto de otro, y es un efecto o lectura de discursos anteriores (VERÓN, 1988a, p.130). La discursividad misma es por él concebida como una red.

Acaso, el corte deba hacerse en términos de transiciones entre espacios mediales o discursivos: el sentido de la circulación no solo está dado por la dimensión del contenido (qué se dice, qué se responde), ni por quiénes son los agentes (quién se comunica con quién: CASTELLS, 2000), sino también por las variantes de sus donaciones y apropiaciones (JENKINS, 2008; 2009; JENKINS, FORD, GREEN, 2013), las inflexiones enunciativas operadas por los tipos de medios involucrados (CARLÓN, 2018), las intersecciones entre circuitos de especializaciones variadas entre distintos tipos de medios (BRAGA, 2017) o las modulaciones que cada superficie mediática ofrece sobre las representaciones y los intercambios (portales de noticias, plataformas de distribución de contenidos culturales, etc.) de acuerdo con lo que los usuarios van haciendo en cada una de ellas (CINGOLANI, 2017).

La circulación en las mediatizaciones contemporáneas es un campo de enorme interés: debemos indagar sus modalidades. Braga propone atender a la conformación de lo que llama circuitos como un modo de ir más allá de las "relaciones directas entre producción y recepción" (BRAGA, 2017, p. 50), considerando las variantes que ofrecen los "medios industriales" (centrales) para generar un espacio propio, pero también de hacerse cargo transversalmente de todos los otros tipos de discursos, o de participar de una interacción con otros alcances (extramediáticas, o incluso provenientes del "sentido común"), descentrándose así como impulsores de iniciativas. No muy 


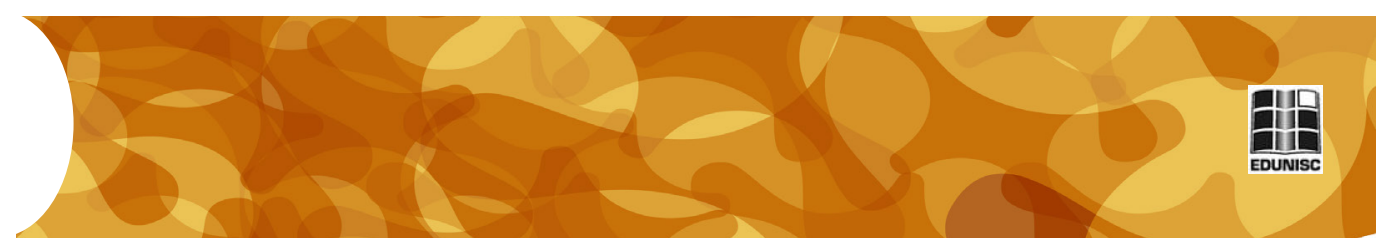

alejado de ese sistema heurístico, Carlón (2017) indaga concretamente en casos de agenda que actualizan nuevos tipos de transiciones (que él llama top-down y bottom-up, según si surgen de los medios centrales o de las redes de interacción inter-individual). Estas transiciones son tipos de circulación que en sí mismas son significantes, es decir, impactan directamente en el sentido del tratamiento público, social, de esos casos. Todos estos trabajos atienden la circulación como tensión entre superficies mediáticas de diferente naturaleza.

Seguramente los estudios se multiplicarán aquí y allá en vistas a las nuevas condiciones de mediatización. No son las llamadas "nuevas tecnologías", tampoco el mercado de los medios ni las variaciones discursivas, los que marcan el escenario próximo, sino una rearticulación de novedades en todos esos niveles a la vez (FERNÁNDEZ, 2008). Habrá que prestar cada vez más atención en el futuro inmediato a los procesos de mayor impacto político y cultural a este tipo de circulaciones.

\section{Referencias}

BATESON, G. Comunicación. In: WINKIN, Y. (comp.) La nueva comunicación. Barcelona: Kairós, 1987.

BATESON, G. Pasos hacia una ecología de la mente: Buenos Aires: Planeta - Carlos Lohlé, 1991.

BRAGA, José Luiz. Circulação \& circuitos: situações. In: CASTRO, Paulo César (org.). A circulação discursiva: entre produção e reconhecimento. Maceió: Edufal, 2017.

CARLÓN, Mario. La cultura mediática contemporánea: otro motor, otra combustión (segunda apropiación de la Teoría de la Comunicación de Eliseo Verón: la dimensión espacial). In: CASTRO, Paulo César (org.). A circulação discursiva: entre produção e reconhecimento. Maceió: Edufal, 2017.

CARLÓN, Mario. Medios individuales, medios colectivos y circulación transversal: desde "adentro hacia afuera" y desde "afuera hacia adentro" (o como afecta la nueva circulación a las instituciones sociales). In: CASTRO, Paulo César (org.). Circulação discursiva e transformação da sociedade. EDUEPB: Campina Grande-PB, 2018, pp. 26-46.

CASTELLS, Manuel. A sociedade em rede. A era da informação: 


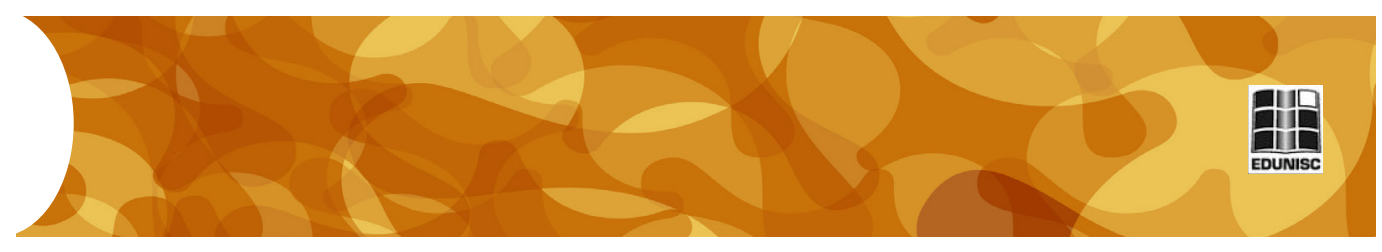

economia, sociedade e cultura. vol. 1, 4. ed. São Paulo: Paz e Terra, 2000 .

CINGOLANI, Gastón. Estrategias para el acceso: los sitios de recomendación como espacios de tensiones en la circulación y mediatización del reconocimiento. In: CASTRO, Paulo César (org.). A circulação discursiva: entre produção e reconhecimento. Maceió: Edufal, 2017, pp. 125-140.

DAYAN, D. (dir.) Hermès 11-12 À la recherche du public. París: CNRS Éditions, 1993.

DAYAN, D. Télévision: le presque-public. Réseaux, v. 18, n. 100, pp. 427-456, 2000. Disponible en: http://www.persee.fr/doc/reso_07517971_2000_num_18_100_2232

FAUSTO NETO, Antônio. A circulação além das bordas. In: VALDETTARO, S. Mediatización, Sociedad y Sentido. Rosario: Dep. de Ciencias de la Comunicación, FCPyRRII, UNR, 2010.

FERNÁNDEZ, J. L. Modos de producción de la novedad discursiva. In: (dir.) La construcción de lo radiofónico. Buenos Aires: La Crujía, 2008.

FERNÁNDEZ, J. L Plataformas mediáticas: Elementos de análisis y diseño de nuevas experiencias. Buenos Aires: La Crujía, 2018.

GARCÍA CANCLINI, Néstor. Consumidores y ciudadanos. Grijalbo: México, 1995.

GROYS, B. Bajo sospecha: una fenomenología de los medios. Valencia: Pre-Textos, 2008.

HALL, Stuart. Encoding/decoding. In: et al. Culture, Media,

Language: Working Papers in Cultural Studies, 1972-79. Taylor \& Francis e-Library, 2005, p. 117-127. [original: 1980]

HANNERZ, U. Cultural Complexity: Studies in the Social Organization of Meaning. New York: Columbia Univ. Press, 1992.

JENKINS, Henry. Convergence Culture: la cultura de la convergencia de los medios de comunicación. Barcelona: Paidós, 2008.

JENKINS, Henry. Fans, blogueros y videojuegos: la cultura de la 


\section{Rizoma}

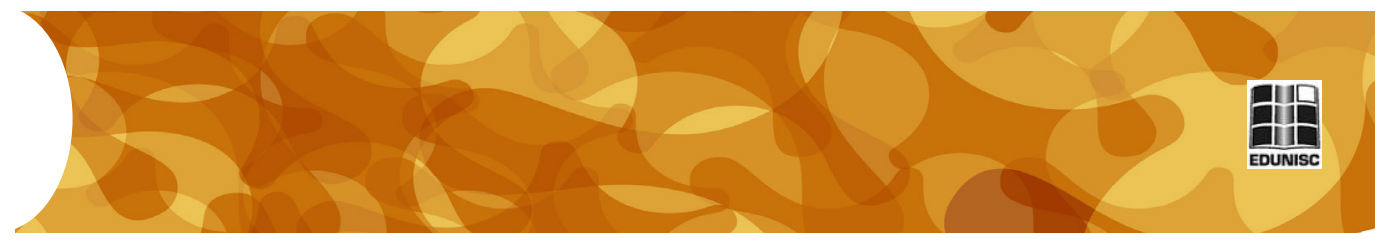

colaboración. Barcelona: Paidós, 2009.

JENKINS, H.; FORD, S.; GREEN, J. Spreadable media: creating value and meaning in a networked culture: New York - London: New York University Press, 2013.

LUHMANN, Niklas. La realidad de los medios de masas. Barcelona - México: Anthropos - Universidad Iberoamericana, 2000.

MARTÍN-BARBERO, Jesus. De los medios a las mediaciones: comunicación, cultura y hegemonía. Gustavo Gili: Barcelona, 1987.

MORLEY, D. Televisión, audiencias y estudios culturales: Buenos Aires: Amorrortu, 1996.

SBARDELOTTO, M. Circulação em rede: a complexa comutabilidade dos polos de produção e recepção no fluxo comunicacional digital. Questões Transversais - Revista de Epistemologias da Comunicação, São Leopoldo, v. 6, nº 11: 48-57, jan./jun. 2018.

SILVERSTONE, R. La moral de los medios de comunicación. Buenos Aires: Amorrortu, 2010.

TRAVERSA, Oscar. Aproximaciones a la circulación discursiva a partir de Eliseo Verón. In: CASTRO, Paulo César (org.). A circulação discursiva: entre produção e reconhecimento. Maceió: Edufal, 2017, pp. 97-108.

VERÓN, Eliseo. Prólogo a la edición española. In: LÉVI-STRAUSS, Claude. Antropología Estructural. Eudeba: Buenos Aires, 1961.

VERÓN, Eliseo. La semiosis social: Fragmentos de una teoría de la discursividad. Barcelona: Gedisa, 1988a.

VERÓN, Eliseo. Presse écrite et théorie des discours sociaux: production, réception, régulation. In: VERÓN, Eliseo. La presse. Produit, production, réception, Paris: Didier Erudition, 1988b. p. 11-25.

VERÓN, Eliseo. Prefacio. In: VERÓN, Eliseo. Conducta, Estructura y Comunicación. 3. edic., Buenos Aires: Amorrortu, 1995.

VERÓN, Eliseo. Les publics entre production et réception: problèmes pour une théorie de la reconnaissance. In: VERÓN, Eliseo. Cursos de Arrábida. Públicos, Televisão, Portugal, 2001. 


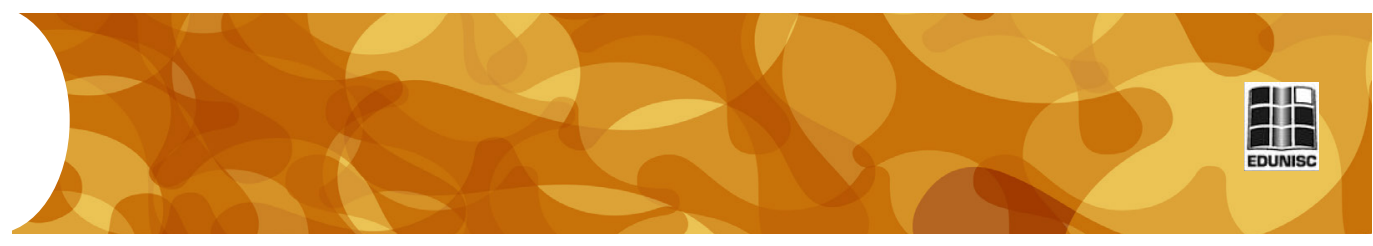

VERÓN, Eliseo. Fragmentos de un tejido. Buenos Aires: Gedisa, 2004.

VERÓN, Eliseo. Informe 2005. Primera parte. Cultura, democracia y desarrollo humano, inédito, 2005.

VERÓN, Eliseo. La semiosis social, 2. Buenos Aires: Paidós, 2013.

VERÓN, E.; SLUZKI, C. Comunicación y neurosis. Buenos Aires: Editorial del Instituto, 1970.

WATZLAWICK, P.; BAVELAS, B.; JACKSON, D. D. Algunos axiomas exploratorios de la comunicación. In: Teoría de la comunicación humana: Interacciones, Patologías y Paradojas. Barcelona: Herder, 1981, pp. 49-71.

WEAVER, W. La matemática de la comunicación. In: SMITH, A. (comp.). Comunicación y cultura, tomo 1 La teoría de la comunicación humana. Buenos Aires: Nueva Visión, 1972.

WIENER, N. Cybernetics. In: SMITH, A. (comp.). Comunicación y cultura, tomo 1 La teoría de la comunicación humana. Buenos Aires: Nueva Visión, 1972.

WINKIN, Y. (comp.) La nueva comunicación. Barcelona: Kairós, 1987. 